\title{
The hidden order behind jerky flow
}

\author{
M.S. Bharathi ${ }^{a}$, M. Lebyodkin ${ }^{\text {b }}$, G. Ananthakrishna ${ }^{\text {a }}$, C. Fressengeas ${ }^{\text {c }}$, \\ L.P. Kubin ${ }^{\text {d,* }}$ \\ ${ }^{a}$ Materials Research Centre, Indian Institute of Science, Bangalore 560 012, India \\ b Institute of Solid State Physics, Russian Academy of Science, 142432 Chernogolovka, Moscow, Russia \\ ${ }^{\mathrm{c}}$ Laboratoire de Physique et Mécanique des Matériaux, Université de Metz-CNRS, Ile du Saulcy, 57045 Metz Cedex 01, France \\ ' Laboratoire d'Etude des Microstructures, CNRS-ONERA 29, Av. de la Division Leclerc, BP 72, 92232 Châtillon Cedex, France
}

Received 30 October 2001; accepted 21 February 2002

\begin{abstract}
Jerky flow, or the Portevin-Le Chatelier effect, is investigated at room temperature by applying statistical, multifractal and dynamical analyses to the unstable plastic flow of polycrystalline $\mathrm{Al}-\mathrm{Mg}$ alloys with different initial microstructures. It is shown that a chaotic regime is found at medium strain rates, whereas a self-organized critical dynamics is observed at high strain rates. The cross-over between these two regimes is signified by a large spread in the multifractal spectrum. Possible physical mechanisms leading to this wealth of patterning behavior and their dependence on the strain rate and the initial microstructure are discussed. (c) 2002 Acta Materialia Inc. Published by Elsevier Science Ltd. All rights reserved.
\end{abstract}

Keywords: Aluminum alloys; Jerky flow; Dynamic phenomena; Chaos; Self-organized criticality

\section{Introduction}

Jerky flow, or the Portevin-Le Chatelier (PLC) effect was discovered as early as the turn of the last century [1]. Since then, it has continuously drawn attention due to its intriguing spatio-temporal dynamics. In this respect, the PLC effect falls into the class of complex non-linear driven systems exhibiting intermittent relaxation sequences, of which there are many examples, including in materials science [2-4]. In uniaxial loading with a constant imposed strain rate, the effect manifests

* Corresponding author. Fax: +33-1-4673-4155.

E-mail address: kubin@onera.fr (L.P. Kubin). itself as serrations in the stress vs. time (or strain) curves, associated with localized bands, static or propagating, of plastic deformation. The phenomenon is observed in many dilute alloys within a definite range of strain rates and temperatures. It is generally agreed that its microscopic origin is the dynamic aging of dislocations by diffusing solute atoms [5-7]. In phenomenological terms, this mechanism results in a negative strain rate sensitivity of the flow stress, which destabilizes uniform plastic flow [8-10].

In single crystals, PLC bands have crystallographic orientations and, in addition, the serrations do not exhibit distinctive features. In contrast, there have been numerous observations showing that polycrystals display well-defined types of ser- 
rations. The latter are traditionally classified into types A, B and C, each associated with a characteristic serration morphology (see Refs. [11-13] for more details and illustration). With increasing strain rate or decreasing temperature, one observes serrations of decreasing amplitude and narrower PLC bands, with an increasing tendency to band propagation. Large non-propagating bands of the type $\mathrm{C}$, which nucleate at random places along the specimen's gauge length, are sometimes found at the smallest strain rates. At intermediate strain rates, type $B$ bands are formed in succession, each nearby the previous one, so that plastic deformation propagates along the sample in a hopping manner. On further increasing the strain rate, band propagation progressively becomes continuous, which characterizes the domain of type A bands. In the last decade, non-local constitutive relations based on various microstructural or mechanical mechanisms leading to a spatial coupling have been employed to tentatively describe these spatiotemporal patterns (see Refs. [14,15] and references therein).

The present work is based on a completely different approach that can be summarized by a simple question. Considering the often complex and seemingly disordered stress vs. time data recorded during jerky flow, is it purely random or does it contain some hidden type of order, from which some physical consequences could be drawn? A preliminary answer to this question was provided by statistical analyses of the stress drops. The first detailed study on single crystals appeared only recently [16]. It showed that the distributions of stress drops shift from bell-shaped at low strain rates, suggesting the existence of characteristic average time and length scales, to asymmetric at high strain rates. In this last case, power-law distributions are obtained, which implies a lack of intrinsic length and time scales. A discrete nonlocal model based on an analogy with a dry friction model for the sliding of crustal faults was able to predict this general trend $[16,17]$.

A more difficult but more revealing way of dealing with the stress vs. time series consists of analyzing their dynamical behavior, as will be shown subsequently. Concepts and methods borrowed from non-linear dynamics can then be used to probe the serrated records. For instance, an underlying order can be due to deterministic chaos, which originates in the dynamic interaction of a few (at last three) variables [18], to self-organized criticality (SOC) which describes avalanche-like behavior with an infinity of degrees of freedom [19], or to more complex regimes. Powerful processing techniques are available for characterizing these dynamical regimes.

Quite unexpectedly, two dynamical regimes were found in the range of existence of the PLC effect. Following an earlier theoretical prediction [20], a first preliminary evidence for deterministic chaos was found in single crystals of copper alloys [21] and soon after in samples of $\mathrm{Al}-\mathrm{Mg}$ alloy [22]. Refined studies confirmed this finding [2324]. In addition, chaotic behavior was shown to correspond to the bell-shaped statistical distributions of stress drops recorded at medium strain rates [24]. Analyses carried out at high strain rates clearly revealed a different dynamics. Scaling relations were found between the power-law exponents drawn from the statistics of the stress drop magnitudes and durations [24]. The underlying dynamical regime was identified as SOC dynamics. In the transition region where the nature of the dynamics changes, a mixture of features from either side is expected for the statistical distributions. In parallel, a spatial heterogeneity of the dynamics is also anticipated. As will be shown subsequently, such heterogeneous conditions are best quantified by a multifractal analysis.

The purpose of this paper is to present new results and a synthesis on the non-linear dynamics of jerky flow in polycrystals, along the lines of a short preliminary study [25]. Connections will be established and discussed between the initial microstructure of the material, the PLC band types, the statistics of the magnitudes of the plastic activity bursts and the newly found types of dynamical regimes. With this aim, a series of experiments on polycrystalline $\mathrm{Al}-\mathrm{Mg}$ samples with different initial microstructures has been carried out over a wide range of strain rates. The present paper is organized as follows. Experimental details on materials, testing and data recording are given in Section 2. Section 3 introduces the relevant types of methodologies and analyses. 
The results are given in Section 4 and the concluding Section 5 provides a discussion of the basic instability mechanisms in the light of these results, emphasizing the fundamental role of dynamical analyses for the understanding of the spatio-temporal aspects of jerky flow.

\section{Experimental}

Three sets of samples were prepared from a polycrystalline cold-rolled sheet of $\mathrm{Al}-2.5 \% \mathrm{Mg}$ alloy. The first set, labeled $r$, consisted of specimens extracted from the as-received material. The grain morphology was anisotropic with an aspect ratio of 5 and an average grain dimension of 30$40 \mu \mathrm{m}$ along the rolling direction. A second set, $a$, was annealed for $4 \mathrm{~h}$ at $593 \mathrm{~K}$ and water-quenched, which lead to a less anisotropic grain structure, with an aspect ratio of 3 and an average grain size of about $50 \mu \mathrm{m}$ in the rolling dimension. The third group of samples, $a a$, was subjected to an additional $3 \mathrm{~h}$ annealing at $733 \mathrm{~K}$ before quenching. This treatment produced a nearly equiaxed structure with a large average grain size of about $1 \mathrm{~mm}$. Flat specimens were machined with their tensile axis parallel to the rolling direction. The tests were carried out at room temperature and consequently, there was only one control parameter, the applied strain rate $\dot{\varepsilon}_{\mathrm{a}}$. To monitor closely its influence on the dynamics of jerky flow, eight values were selected in the range from $5.56 \times 10^{-6} \mathrm{~s}^{-1}$ to $1.39 \times 10^{-2} \mathrm{~s}^{-1}$ (cf. Table 1 ). The PLC effect was observed throughout this range.

The stress vs. time response was recorded electronically at periodic time intervals. The selection of the sampling interval plays a crucial role in subsequent data processing. In order to obtain meaningful results, the recording frequency must be large enough to sample with at least a few data points the fastest events, viz. the serrations. On the other hand, too high sampling frequency would lead to unnecessarily large data sets. As a result, a sampling rate of $20 \mathrm{~Hz}$ was selected, except at the highest applied strain rate where a $200 \mathrm{~Hz}$ frequency was used. Statistics must be sampled on data sets that do not show significant drift associa- ted with microstructural evolution and strain hardening. The experimental stress vs. strain curves often displayed a transient stage with strong hardening at small strains $(\varepsilon<5 \%)$, followed by a stage where strain hardening progressively saturates. Therefore, the data sets were recorded at large enough strains $(\varepsilon \approx 15 \%)$ in the quasi saturated regime, over strain intervals of typically $2-$ $5 \%$. The total number of recorded data points is typically 10,000 up to $5.56 \times 10^{-3} \mathrm{~s}^{-1}$ and 3500 5000 beyond this value. In addition, a systematic increase in the average size of the stress drops was observed with increasing deformation, a rather common feature of the PLC effect. The average stress drop size was normalized and leveled off by using a moving average procedure. The investigations presented below were carried out on the resulting time series. Three typical corrected stress-time curves are shown in Fig. 1(a), (c) and (e) for three different strain rates. Fig. 1(b), (d) and (f) shows the corresponding distributions of the amplitudes of the plastic activity bursts, i.e. the time derivative of the stress (in absolute values). This quantity better reflects the dynamics of jerky flow than the stress drop values used in previous investigations and was found more suitable for performing statistical analyses.

\section{Methodology}

It is now established that the apparent randomness of a time series could result from a purely deterministic evolution of a system with a few degrees of freedom. This is called deterministic chaos. Then, the hidden order can be recovered by unfolding the original multidimensional dynamics from the experimental one-dimensional time series by reconstructing a phase space of appropriate dimension. Such a reconstruction is always possible if the time series is reasonably long and noise free [26]. Further analysis is then applied to the characterization of the orbit of the system in its phase space. The complexity and apparent randomness in the time evolution of the observed variables stem, in the first place, from its sensitivity to initial conditions, which leads to a progressive loss of memory of the initial state. This effect is 

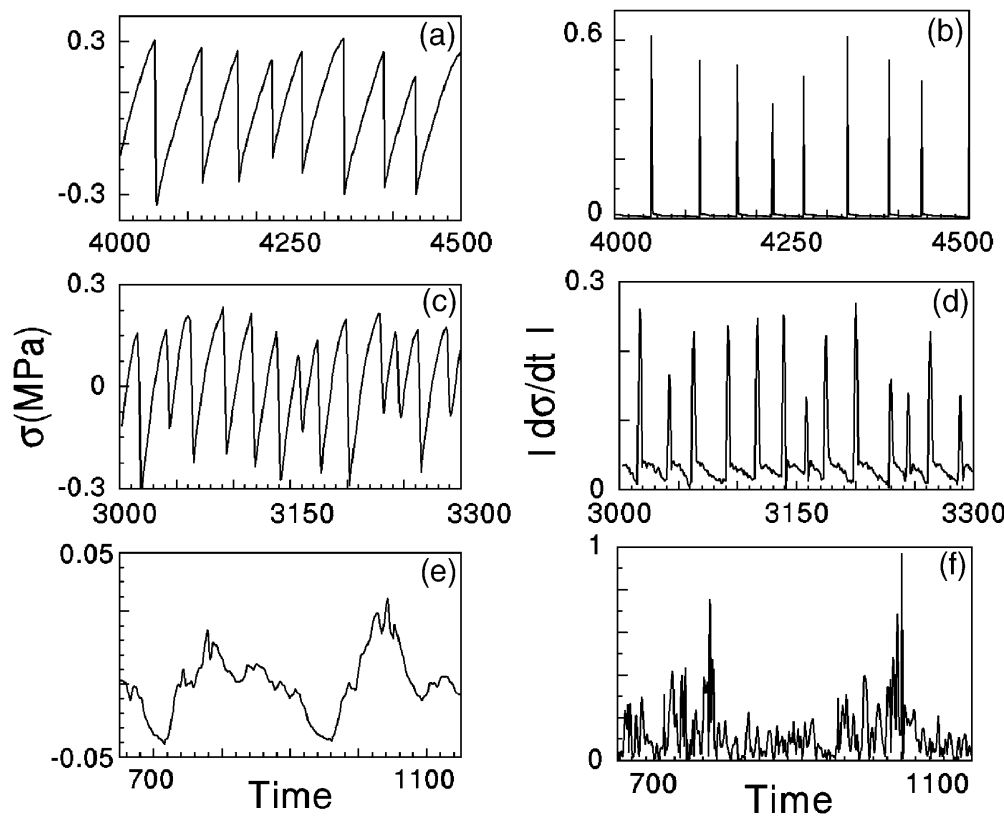

Fig. 1. Left: time series data for the (as-received) $r$ microstructure, with increasing strain rate from top to bottom: $\dot{\varepsilon}_{a}=$ $5.56 \times 10^{-6}, \dot{\varepsilon}_{a}=2.78 \times 10^{-4}$ and $\dot{\varepsilon}_{a}=5.56 \times 10^{-3} \mathrm{~s}^{-1}$ (files $r 1, r 4, r 7$, respectively). Right: the corresponding absolute values of the derivative of stress with respect to time.

reflected by the local exponential divergence in some directions (stretching directions) of neighboring orbits in the phase space. This divergence is measured, in each direction and in average over the whole phase space, by the so-called Lyapunov exponents. Folding of the orbits in other directions must also occur since, in our dissipative driven system, elementary volumes in the phase space are contracting. Along one particular direction, which is tangential to the orbit, there is neither contraction nor expansion, i.e. the corresponding Lyapunov exponent is zero. As a result of stretching and folding, the orbit exhibits self-similar properties and organizes itself into a geometrical object of fractal dimension $D_{\mathrm{f}}$, called a strange attractor.

The reconstruction of the multidimensional dynamics starts from the time series $\{\sigma(k), k=$ $1,2, \ldots, K\}$, where $k$ is in units of the sampling time and $K$ is the number of data points. This scalar set is unfolded into a $d$-dimensional space by introducing the vectors

$$
\begin{aligned}
& \vec{\xi}_{k}=[\sigma(k), \sigma(k+\tau), \ldots, \sigma(k+(d-1) \tau], \\
& k=1, \ldots,(K-(d-1) \tau)
\end{aligned}
$$

where $\tau$ is the delay time [27]. The vectors $\left\{\xi_{k}, k=1, \ldots, K-(d-1) \tau\right\}$ define points on the orbit followed in the reconstructed phase space. When applying the reconstruction process to experimental data, a key issue to be addressed is what time lag $\tau$ and dimension $d$ are to be chosen for a proper description of the dynamics [15]. In order to characterize the fractal nature of the attractor one usually employs, instead of the fractal dimension $D_{\mathrm{f}}$, the correlation dimension $v$, which can be conveniently determined from the Grassberger-Procaccia algorithm [28]. In this method, the correlation integral $C(r)$ is defined as the fraction of the pairs of vectors $\left(\vec{\xi}_{i}, \vec{\xi}_{j}\right)$ whose distance is less than a specified value $r$. The selfsimilar structure of the attractor, when it exists, is reflected in a scaling relation $C(r) \propto r^{v}$ in the limit of small $r$. As the embedding dimension $d$ is increased, the slope $\ln C(r) / \ln r$ tends to a constant value taken as the correlation dimension. Although care must be exercised [29], the smallest integer larger than $v+1$ reflects the number of modes required to describe the dynamics. Finally, the 
spectrum of Lyapunov exponents of the attractor is computed using an algorithm by Eckman et al. [30], suitably modified for short time series (see Ref. [24]). The domain containing the neighbors

of a reference vector $\vec{\xi}_{i}$ is taken as a shell, in order to remove noise-affected close neighbors. The outer radius of the shell is taken as large as needed for proper sampling of the statistics. We consider the dynamics to be deterministic if a fair range of the shell size $\varepsilon_{\mathrm{s}}$ can be found such that stable values, i.e. constant in that range, emerge for a positive exponent and a zero exponent [24] (cf. Fig. 3).

For a chaotic dynamics, the orbit can be described by a small number of degrees of freedom. In the case of the PLC effect, these variables have been identified as different types of dislocation densities, along with the applied stress [20]. In contrast, for a self-organized critical dynamics (and also for random systems), the orbit fills up an infinite dimensional phase space when $K$ tends to infinity. Similarly, the statistical distribution of events $p(x)$ ( $x$ being typically the magnitude of plastic activity bursts or their duration) exhibit power-law behavior, e.g. $p(x) \propto x^{-a}$, which implies lack of intrinsic time and length scales. Indeed, the functional relation $p(k x) \propto k^{-a} p(x)$ displays scaling symmetry, meaning that the distribution is selfsimilar in this scaling operation. The term 'selforganized' appearing in SOC refers to the propensity of spatially driven systems (here the tensile sample) with a built-in threshold for bursts of plastic activity to spontaneously display (deformation) patterns at all length scales. The reference to 'criticality' points at the lack of a characteristic scale and, therefore, the existence of long-range spatial correlations, as in the case of systems undergoing second order phase transitions close to a critical point [31].

Multifractal analysis can be introduced from the point of view of chaotic dynamics. Since the strange attractor is a fractal with fractal dimension $D_{\mathrm{f}}<d$, it does not fill the $d$-dimensional reconstructed phase space. Thus, it is appropriate to consider the number of times, denoted $n_{\mathrm{j}}$, an orbit visits the $j$ th $d$-dimensional cube of linear dimension $\delta r$. The probability of visiting that cube is $p_{j}(\delta r)=n_{j} / \sum_{k=1}^{N} n_{k}$, where $N=N(\mathrm{~d} r)$ is the number of cubes required to cover the attractor. If the latter is a conventional homogeneous fractal, it is adequately described by an exponent, and its frac-

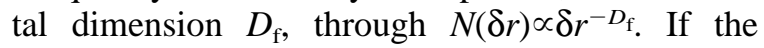
attractor is inhomogeneous, different parts have different visiting probabilities and the fractal dimensions corresponding to these different regions are different. The probabilities $p_{j}(\delta r)$ then scale as $p_{j}(\delta r) \propto \delta r^{\alpha}$, with the parameter $\alpha$ taking a continuum of values corresponding to the different subsets [32]. The exponent $\alpha$ goes by the name of singularity index or singularity strength. Then, one generalizes the relation $N(\delta r) \propto \delta r^{-D_{\mathrm{f}}}$ to $N_{\alpha}(\delta r) \propto \delta r^{-f(\alpha)}$, where $N_{\alpha}(\delta r)$ is the number of cubes required to cover the subset of singularity strength $\alpha$, and $f(\alpha)$ its fractal dimension. The more non-uniform the attractor, the larger the range of $\alpha$ values [32]. The non-uniform nature of the set is then captured by the range of multifractality (MF) $\theta=\alpha_{\max }-\alpha_{\min }$, where $\alpha_{\max }$ and $\alpha_{\min }$ are the extreme values of $\alpha$. Although this presentation utilizes a chaotic strange attractor, the heterogeneous nature of the object may emerge from other types of complex dynamics as well. In the present work, the simplest heterogeneous set was used, namely one-dimensional records of the absolute magnitude of stress-time series derivative, $|\mathrm{d} \sigma / \mathrm{d} t|$, representing the plastic activity bursts. For a computation of the relevant quantities, the reader is referred to Refs. [32,33].

\section{Results}

Table 1, which will be further commented, summarizes the results of the present investigations. For each initial specimen condition (cold-rolled, annealed and doubly annealed), and as a function of strain rate, the upper line indicates the dynamic regime (chaos, self-organized criticality) or an intermediate region of varying degree of MF. The lower line indicates the type of distribution of the plastic activity bursts: peaked (P), double peaked (DP), broadly asymmetric (BAS) or of power-law type (PL). The type of associated PLC bands is also indicated (A/B refers to a mixed mode of type A/type B bands). 


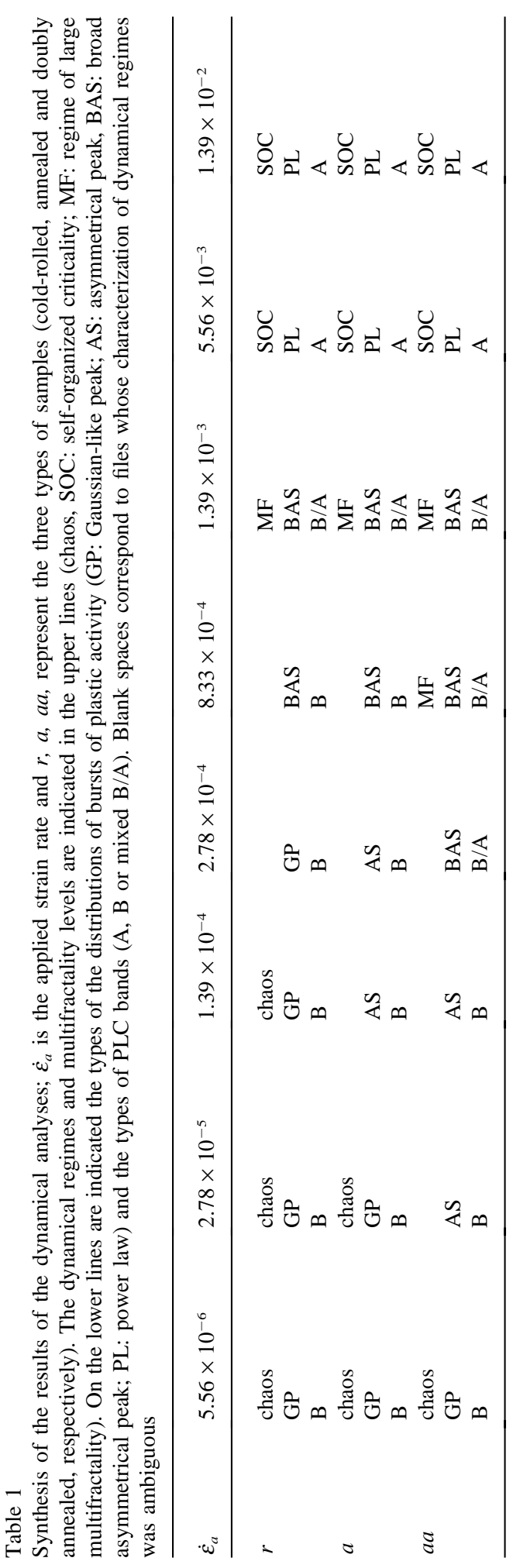




\subsection{Dynamical analyses}

We start with the results of the dynamical analyses at low and medium strain rates. Both the correlation dimension and Lyapunov spectrum were calculated. Positive results in terms of chaotic dynamics were obtained from all the three sets of samples in a definite range of strain rates. Since the results are all similar, they are illustrated with the data file $r l$ from the as-received sample at the lowest strain rate (the files are numbered by order of increasing strain rate value).

The correlation integrals $C(r)$ were calculated using a delay time $\tau=8$ sampling units (for this file). The result is shown in Fig. 2 for the embedding dimensions $d=15-18$. A scaling region can be seen in the interval $-4.9<\ln r<-3.2$, with the slopes converging as the embedding dimension approaches $d=18$. The best linear fit for the resulting correlation dimension yields $v \approx 4.15$ (in Ref. [25], data were shown on file $r 1$, but the value quoted in the text, $v \approx 4.6$, unfortunately referred to file al). The smallest integer larger than $v+1$ being 6 , the system dynamics should result from the non-linear interaction of six modes. The Lyapunov spectrum was calculated for the embedding dimension $d=6$ (Fig. 3). Stable positive and zero exponents were found in the range $7 \%<\varepsilon_{\mathrm{s}}<$ $12 \%$, where $\varepsilon_{\mathrm{s}}$ is the size of a shell sampling the

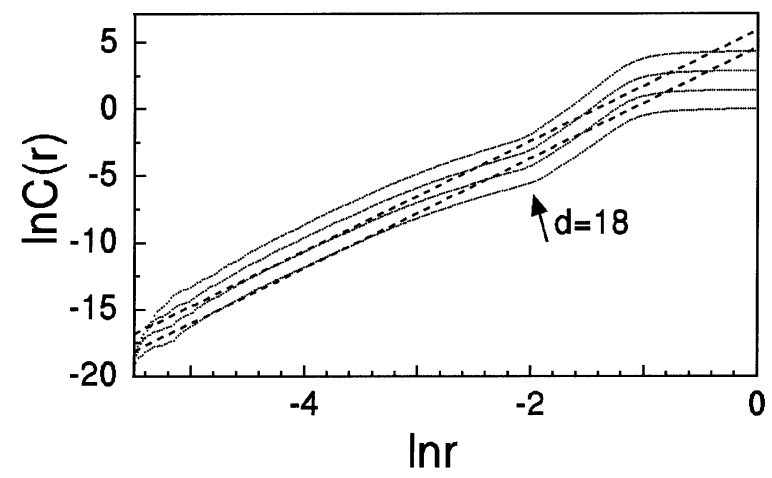

Fig. 2. File $r 1$. Correlation integral $C(r)$ using embedding dimensions $d=15-18$ and a delay time $\tau=8$, for $\dot{\varepsilon}_{a}=$ $5.56 \times 10^{-6} \mathrm{~s}^{-1}$. For the sake of clarity, the curves corresponding to $d=15-17$ have been translated upwards with respect to $d=18$ by a constant amount. Dashed lines are shown as guide to the eye. The slopes converge towards a constant value for $d=18$, defining a correlation dimension $v=4.15$. data points and measured in percent of the attractor size. A consistency check consists of calculating with the help of the Kaplan-Yorke conjecture [36], the Lyapunov dimension $D_{\mathrm{KY}}$, which should be close to the correlation dimension $v$. The value calculated from the Lyapunov spectrum of Fig. 3 turns out to be $D_{\mathrm{KY}} \approx 4.2 \approx v$. Other consistency checks such as the surrogate data analysis (see Ref. [29] for a discussion of this method) were successfully performed, confirming that the time series is indeed of chaotic nature.

Similar positive results, with same value of the correlation dimension, were obtained for the coldrolled samples (files $r 2$ and $r 3$ ) and for the annealed samples within a smaller range of strain rate. For the strongly annealed microstructure, evidence of chaos was only obtained at the lowest strain rate. Therefore, as shown in Table 1, a chaotic region has been found in a domain of strain rates that shrinks towards low values with increasing loss of memory of the cold-rolled state. This domain was found to coincide with one of Gaussian-like distributions for bursts of plastic activity. Whereas chaos is always associated with type B bands, its unambiguous identification becomes impossible as soon as the distributions of stress drop rates become asymmetrical, even if the PLC bands still look like type B bands. As will be seen subsequently, this is due to a departure from chaotic behavior towards a weak multifractal regime.

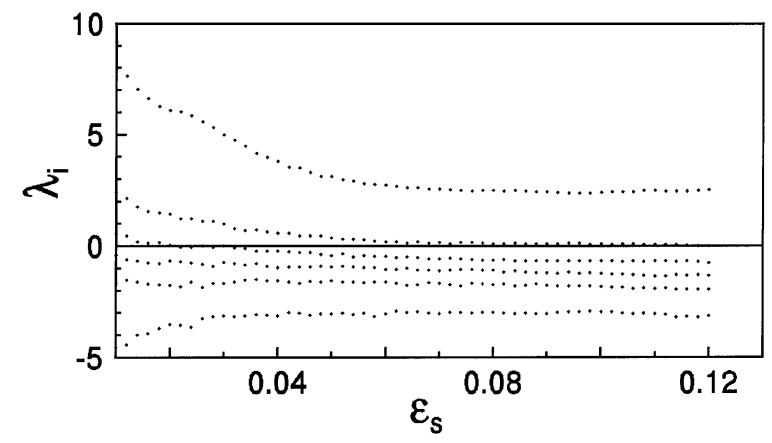

Fig. 3. Spectrum of Lyapunov exponents $\lambda_{\mathrm{i}}$ for $\dot{\varepsilon}_{a}=$ $5.56 \times 10^{-6} \mathrm{~s}^{-1}$, as a function of the size of the shell, $\varepsilon_{\mathrm{s}}$, used to sample the data points. The embedding dimension is $d=$ 6. The occurrence of one positive and one zero value, stable over a range of $\varepsilon_{\mathrm{s}}$, confirms that the behavior is chaotic. 


\subsection{Statistical analysis}

As indicated above, the plastic activity bursts are represented by the modulus of the time derivative of $\sigma(t),|\mathrm{d} \sigma / \mathrm{d} t|$. We refer to the finite difference approximant of this quantity by the generic notation $\Psi$, and use this time-dependent quantity for statistical and multifractal analyses. As an illustration, Fig. 1 shows, in addition to three typical stress time series, the corresponding values of $\Psi$. Since the results of the statistical analysis are essentially the same in all types of microstructure, they are illustrated here with the as-received set $r$. The distributions $p(\Delta \Psi)$ and $p(\Delta t)$ of the magnitudes $\Delta \Psi$ and durations $\Delta t$ of the bursts are investigated. Plots of $p(\Delta \Psi)$ are shown in Fig. 4, illustrating how the distributions evolve with applied strain rate (see also Table 1). Peaked distributions are seen in the chaotic regime, indicating the existence of characteristic values. As $\dot{\varepsilon}_{\mathrm{a}}$ is increased, the distributions acquire another peak in the mid-region of $\Delta \Psi$. With further increase in $\dot{\varepsilon}_{\mathrm{a}}$ these two peaks tend to merge, resulting in power-law distributions $p(\Delta \Psi) \approx \Delta \Psi^{-a}$ (cf. Fig. 4(c)). For the data set $r 7$, the scaling exponent is $a \approx 1.5$ over more than one order of magnitude.

Similarly, the distributions of the burst durations are also found to be conveniently fitted with a power-law $p(\Delta t) \propto \Delta t^{-b}$, with $b \approx 3.2$. In addition, the conditional average $\langle\Delta \Psi\rangle_{c}$ (i.e. the mean of $\Delta \Psi$ at time $t$, given information at time $(t-\Delta t))$ for a given value of $\Delta t$ scales as $\Delta t^{x}$ with $x \approx 4$.2. Thus, the scaling exponents verify the relation $x(a-$ 1) $+1-b=0$ quite well. This relation was shown to be compatible with SOC dynamics [34].

Table 1 summarizes the results for all investigated files, showing the region where SOC-type dynamics is found. This region coincides with the domain of high strain rates where the propagating type A bands are observed. At low strain rates, it is bounded by a transition region to chaotic dynamics. The transition domain progressively extends to low strain rates when going from cold-rolled to doubly annealed specimens. In this range, the distributions $\Delta \Psi$ are rather large (cf. Fig. 4(b)), and are better characterized by a multifractal approach.

\subsection{Multifractal analysis}

The multifractal analysis has been carried out on all samples and in the entire range of strain rates. In every case, the range over which $\alpha$ and $f(\alpha)$ were calculated is at least one order of magnitude in $\Delta t$. A plot of $f(\alpha)$ for the file $r 6$ corresponding to the strain rate where the extent of $\alpha$ is maximum, is shown in Fig. 5. Notice that the maximum value of $f(\alpha)$ is 1 , as it should be for a one-dimensional set. Such a result is usually considered a good indicator of the accuracy of the calculations.

The range of MF $\theta$ is taken as an order parameter, and its dependence on the driving parameter $\dot{\varepsilon}_{a}$ is shown in Fig. 6 for all three types of microstructures. For both the $r$ and $a$ files, it is seen to keep relatively low values at both low and high
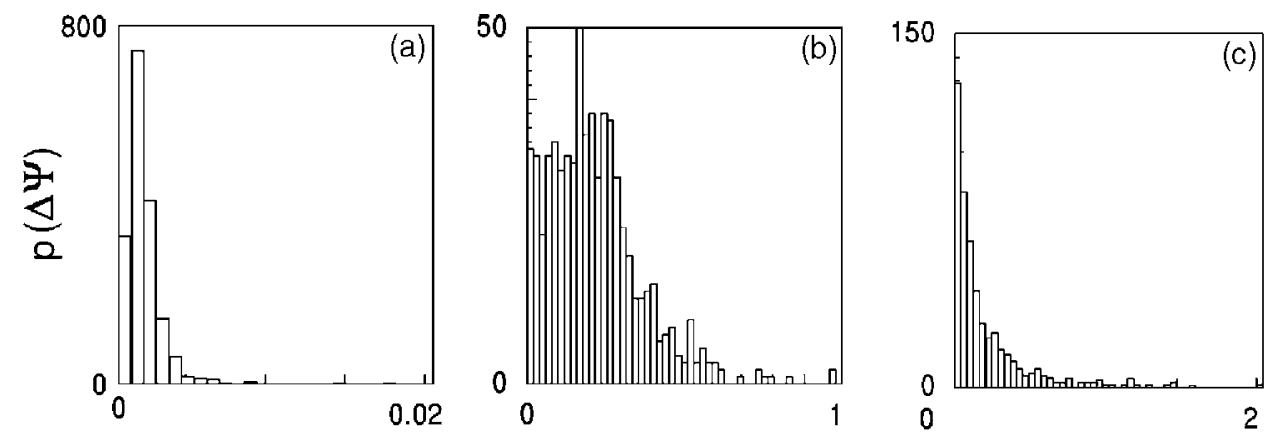

$\Delta \Psi$

Fig. 4. Distribution $p(\Delta \Psi)$ of the amplitude of the stress rate bursts (absolute values) for the data sets at (a) $\dot{\varepsilon}_{a}=5.56 \times 10^{-6} \mathrm{~s}^{-1}$, (b) $\dot{\varepsilon}_{a}=1.39 \times 10^{-3} \mathrm{~s}^{-1}$ and (c) $\dot{\varepsilon}_{a}=5.56 \times 10^{-3} \mathrm{~s}^{-1}$ (files $r l, r 6$ and $r 7$, respectively). Notice the evolution from peaked to broadly asymmetric and monotonously decreasing distributions with increasing strain rate. 


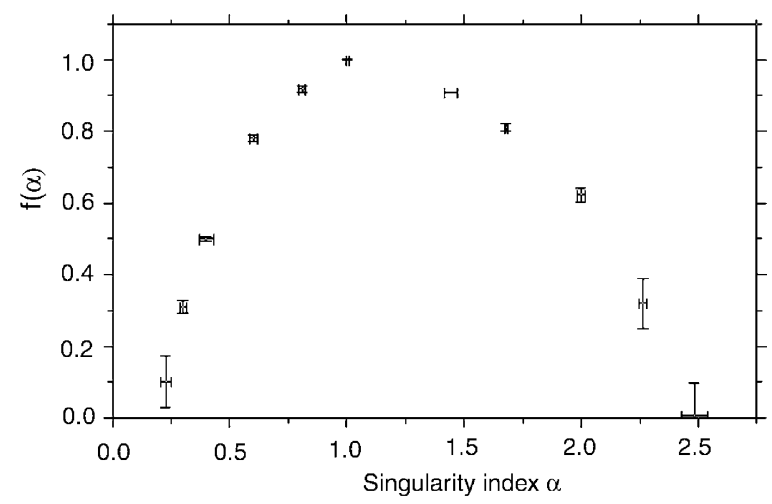

Fig. 5. Multifractal spectrum $f(\alpha)$ for the data set at an applied strain rate $\dot{\varepsilon}_{a}=1.39 \times 10^{-3} \mathrm{~s}^{-1}$ (file $r 6$; error bars are indicated). The inverse parabolic shape is typical for multifractal entities. Notice the extent of the singularity strength values $\alpha$ from $\alpha_{\min }=0.2$ to $\alpha_{\max }=2.5$. This value of the multifractal range $\theta=\alpha_{\max }-\alpha_{\min }$ is carried over in Fig. 6 .

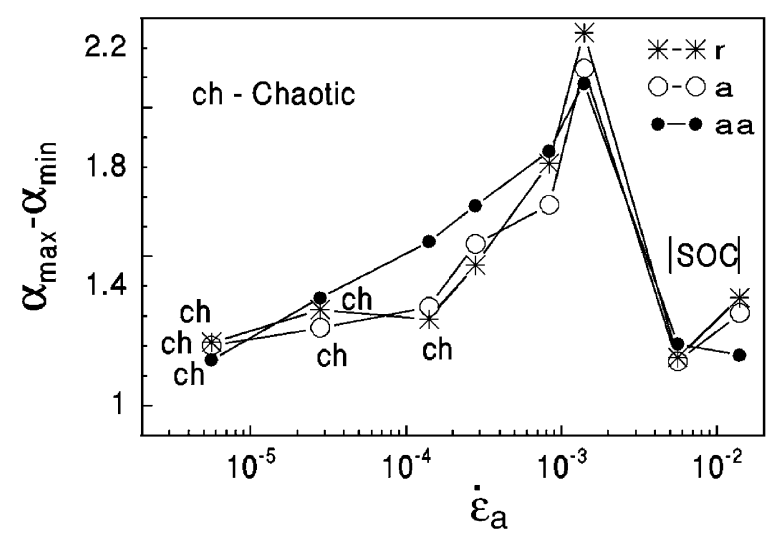

Fig. 6. The multifractal range $\theta=\alpha_{\max }-\alpha_{\min }$ as a function of applied strain rate $\dot{\varepsilon}_{a} . \theta$ keeps relatively low values at both low and high strain rates. In contrast, a sharp peak is observed at intermediate values of strain rate, signaling a transition from chaotic to SOC-like behavior (the corresponding PLC band types are given in Table 1). The high level of multifractality in this region (files $r 6, a 6, a a 6$ ) reflects the spatial heterogeneity of the plastic activity.

strain rates. In the chaotic regime, small values of the order parameter can be traced to the existence of a sharp peak in the distribution of the stress drop amplitude. We also note that at low and medium strain rates, the values of $\theta$ for the $a a$ samples are generally lying substantially above those for the $a$ and $r$ samples. This reflects the fact that asymmet- ric distributions are seen at relatively small values of the strain rate for the $a a$ samples, compared to the other samples. This should also be anticipated from the fact that chaos cannot be detected beyond the first strain rate value. In the region of SOC, small values of $\theta$ are due to the scaling distribution occurring at high strain rates (Fig. 4(c)) [32]. In contrast, a sharp peak in the order parameter is observed at intermediate values of strain rate, which clearly signals a transition in the nature of dynamics from chaotic to SOC type. The high level of MF in this region reflects the spatial heterogeneity of the plastic activity. The multifractal analysis confirms that the extent of the chaotic regime is microstructure sensitive (cf. Table 1 and Fig. 6), whereas the domain of SOC is only slightly affected and seems more intrinsic. As a consequence, the transition gap between chaos and SOC regions is also structure sensitive and increases with annealing.

\subsection{Comparison with single crystal data}

While no new single crystal data is presented here, it appears worthwhile to recall single crystal results for the sake of comparison with the present polycrystalline data. In $\mathrm{Cu}-\mathrm{Al}$ single crystals oriented for single glide, the correlation dimension was found to be $v \approx 2.7$ at low strain rates [24], thus significantly smaller than the value $v \approx 4.15$ measured in the present study. The implication is that less degrees of freedom are required in single crystals for the description of the dynamics of jerky flow. That less dynamical complexity is displayed by single crystals comes as no surprise, but the comparison of the numbers confirms the consistency of the dynamical analysis.

A SOC regime found at high strain rates in single crystals [24] had exponent values typically around unity, which is again much lower than the present polycrystalline values. The faster decay of distributions in polycrystals implies, by comparison with single crystals, smoother evolutions in time and a smaller range of time scales involved in the dynamics. 


\section{Discussion and conclusions}

The PLC effect offers a fascinating example of an easily controllable system where such complex dynamical regimes as chaos and SOC are both observed at the price of a simple adjustment of the experimental conditions. The PLC effect is peculiar in the sense that, due to huge collective effects playing the role of a magnifying lens, the dynamical analysis of deformation tests on bulk samples can be used to gain insight into collective defect properties. However, the notion that dislocations exhibit chaotic or SOC dynamics may apply as well to macroscopically uniform plastic flow. This conjecture can be checked by monitoring variables more sensitive to local bursts of dislocation activity than the applied stress or the average strain. For instance acoustic emission data from ice crystals deformed in creep have recently been analyzed, leading to the identification of power-law distributions and possibly SOC dynamics [35].

In the present study, a correspondence between the distribution of plastic activity bursts, the band types and the types of dynamics has been established for $\mathrm{Al}-\mathrm{Mg}$ polycrystals. For $\mathrm{Cu}-\mathrm{Al}$ single crystals, the same correlation was already known to exist between the stress drop distributions and the dynamical regimes [24]. Thus, the pattern of behavior seems to be fairly general. In polycrystals, the chaotic regime is associated with hopping type B bands. It is obtained in a structure-sensitive range of strain rates. In the upper range of strain rates corresponding to type $\mathrm{B}$ bands, asymmetrical distributions of bursts of plastic activity and an increased level of MF signal the extent of the transition region between chaos and SOC. In that region, no unambiguous characterization of the dynamic regime is possible, due to increasing heterogeneity. Present at low strain rates in all microstructures, chaos invades higher strain rates as the material is microstructurally hardened. As chaotic behavior is conveniently described by the evolution of a small number of coupled degrees of freedom including dislocation densities, the sensitivity to microstructure is no surprise. In contrast, the range of SOC dynamics and type A propagating bands is not structure sensitive, which can be understood from the far-reaching long-range corre- lation and the lack of a characteristic scale associated with SOC. As a consequence, the width of the transition domain between chaos and SOC increases with decreasing applied strain rate and material flow stress. MF provides a measure of the heterogeneity of the dynamics in this region, in the form of a strong burst in the order parameter $\theta$ at the cross-over between the high and low strain rate regimes.

A possible approach for understanding the observed types of dynamics consists of considering the properties of three relevant times or length scales. The interplay of these characteristic scales and their variations with the loading rate and the microstructure are the key issues to be clarified. The characteristic loading time between two serrations, $t_{\mathrm{L}}$, is a complex quantity because the reloading sequences are not purely elastic. Nevertheless, it globally decreases when the loading rate increases. The strain gradients occurring during jerky flow can be interpreted in different manners, leading to different models for the spatial coupling term that enters the spatio-temporal constitutive formulations. To fix the ideas, we adopt here the interpretation provided in Ref. [17], but the present arguments are fully general. The spatial coupling is assumed to originate in the elastic internal stresses arising from geometric incompatibilities between differently strained regions, both in single and polycrystals. Plastic relaxation and recovery of these internal stresses may occur with a characteristic time $t_{\mathrm{R}}$ if sufficient time is allowed, which leads to a decreasing intensity of the spatial coupling. Finally, a correlation length scale $l_{\mathrm{P}}$ is defined, which is essentially the distance over which internal stresses in the strained regions can contribute to the activation of slip in the non-deformed ones. This length scale decreases with increasing relaxation of the spatial coupling.

The spatially uncorrelated type $\mathrm{C}$ bands and serrations have not yet been the object of any quantitative investigation. The lack of spatial correlation is simply due to the fact that, at low strain rate, the loading time is much larger than the relaxation time: $t_{\mathrm{L}} \gg t_{\mathrm{R}}$, so that the correlation length scale is vanishingly small. The strain gradients produced by a previous band are relaxed below the level of internal stress fluctuations along the specimen 
gauge length. As the applied strain rate increases, less time is allowed for plastic relaxation to occur. The nucleation of a band happens to be favored within the finite correlation distance $l_{\mathrm{P}}$ of the previous band, hence the hopping aspect of the plastic area spreading. In this domain, the spatial correlation is non-zero but, as its range of influence is limited, it only governs band initiation. From the clear separation of events, which contrasts with the situation at higher strain rates, one does not expect a large number of degrees of freedom in the dynamics. The occurrence of chaos seems natural, although it is not predictable with such simple arguments as the present one.

At high loading rates, very little or no plastic relaxation can occur during the reloading time. Further, the amplitude of the stress drops decreases and becomes vanishingly small. Thus, the stress is always close to the critical value for the onset of a plastic burst, as expected in a SOC system. New bands form before plastic relaxation is complete, which results in a recurrence of partial relaxation events. Hence, a hierarchical distribution of plastic activity bursts occurs, leading to the power-law distributions without a characteristic value associated with SOC dynamics. In parallel, type A bands propagate continuously due to a large correlation length scale $l_{\mathrm{P}}$ and due to the small strain in the bands, whose plastic activity saturates very fast under the effect of strain hardening. The cross-over regime in the dynamics is expected when the reloading time $t_{\mathrm{L}}$ is comparable to the plastic relaxation time $t_{\mathrm{P}}$. The main conclusion from this qualitative discussion is that a valuable spatio-temporal model for the PLC effect must include the local internal stresses, which are usually modeled via a Laplacian term, but also by a time-dependent mechanism for their relaxation. As was shown in Ref. [17], it is possible, by modifying the amplitude of the spatial coupling, to reproduce all types of bands and statistics, as well as the influence of the initial microstructure. Although this has not been checked to date, the corresponding dynamic regimes should also be reproduced by such types of models.

The comparison between single and polycrystal behavior also raises interesting questions. In the domain of deterministic chaos, the correlation dimension and number of degrees of freedom are increased from single to polycrystals (cf. section 4.2). This is likely to be due to a transition between planar, crystallographic single slip in single crystals to the three-dimensional and multislip nature of polycrystal plasticity. In the domain of SOC and propagating bands, since the values of exponents are higher in the case of polycrystals, the consequence is a more regular temporal evolution of the stress drops than in single crystals. One may speculate that this feature is the result, in polycrystals, of a smaller scale in the dislocation microstructure, since the stress is comparatively larger, a better spatial uniformity of the local stresses at distances larger than the grain size and perhaps a smaller sensitivity to surface condition is found.

\section{Acknowledgements}

Support from CNRS and JNCASR under PICS program 657 is gratefully acknowledged. G.A. and M.L. acknowledge the support of University of Metz for several stays in the period 1998-2000. M.L. would like to acknowledge the support of the Russian Foundation for Basic Research and G.A. that of the Department of Science and Technology, India.

\section{References}

[1] Portevin A. Rev Metall 1909;6:914.

[2] Bowden FD, Tabor D. The friction and lubrication of solids, Part I. Oxford: Clarendon Press, 1950.

[3] de Gennes PG. Rev Mod Phys 1985;57:827.

[4] Bak P, Tang C. J Geophys Res 1989;94:16636.

[5] Cottrell AH. Dislocations and plastic flow in crystals. London: Oxford University Press, 1953.

[6] Friedel J. Dislocations. Oxford: Pergamon Press, 1964.

[7] Van den Beukel A. Phys Status Solidi A 1975;30:197.

[8] Penning P. Acta Metall 1972;20:1169.

[9] Kocks UF. In: Progress in materials science, 18. Oxford: Pergamon Press; 1981. p. 18-5.

[10] Kubin LP, Estrin Y. Acta Metall 1985;33:397.

[11] Brindley BJ, Worthington PJ. Metall Rev 1970;145:101.

[12] Pink E, Grinberg A. Mater Sci Eng 1981;51:1.

[13] Chihab K, Estrin Y, Kubin LP, Vergnol J. Scripta Metall 1987;21:203.

[14] Zaiser M, Hähner P. Physica Status Solidi B 1997;199:267. 
[15] Kubin LP, Fressengeas C, Ananthakrishna G. In: Nabarro FRN, Hirth JP, editors. Dislocations in solids, vol. 11. Amsterdam: Elsevier Science, in press.

[16] Lebyodkin M, Bréchet Y, Estrin Y, Kubin LP. Phys Rev Lett 1995;74:4758.

[17] Lebyodkin M, Dunin-Barkowskii L, Bréchet Y, Estrin Y, Kubin LP. Acta mater 2000;48:2529.

[18] Bergé P, Pomeau Y, Vidal C. Order within chaos, towards a deterministic approach to turbulence. New York: Wiley, 1984.

[19] Bak P, Tang C, Wiesenfeld K. Phys Rev A 1988;38:364.

[20] Ananthakrishna G, Valsakumar MC. J Phys D 1982;15:L171.

[21] Ananthakrishna G, Fressengeas C, Grosbras M, Vergnol J, Engelke C, Plessing J, Neuhauser H, Bouchaud E, Planès J, Kubin LP. Scripta Metall Mater 1995;32:1731.

[22] Venkadesan S, Murthy KPN, Rajasekhar S, Valsakumar MC. Phys Rev E 1996;56:611.

[23] Noronha SJ, Ananthakrishna G, Quaouire L, Fressengeas C, Kubin LP. Int J Bifurcat Chaos 1997;7:2577.

[24] Ananthakrishna A, Noronha SJ, Fressengeas C, Kubin LP. Phys Rev E 1999;60:5455.
[25] Bharathi MS, Lebyodkin L, Ananthakrishna G, Fressengeas C, Kubin LP. Phys Rev Lett 2001;87:165508.

[26] Ding M, Grebogi C, Ott E, Sauer T, Yorke JA. Phys Rev Lett 1993;70:3872.

[27] Packard NH, Crutchfield JP, Farmer JD, Shaw RS. Phys Rev Lett 1980;45:712.

[28] Grassberger P, Procaccia I. Physica D 1983;9:189.

[29] Theiler J, Eubank S, Longtin A, Galdrikian B, Farmer JD. Physica D 1992;58:77.

[30] Eckmann JP, Kamphorst SO, Ruelle D, Ciliberto S. Phys Rev A 1986;34:4971.

[31] Bunde A, Havlin S. Fractals and disordered systems. Berlin: Springer, 1991.

[32] Halsey TC, Jensen MH, Kadanoff LP, Procaccia I, Shraiman BI. Phys Rev A 1986;33:1141.

[33] Chhabra AB, Jensen RV. Phys Rev Lett 1989;62:1327.

[34] Kertész J, Kiss LB. J Phys A 1990;23:L433.

[35] Miguel M-C, Vespignani A, Zapperi S, Weiss J, Grasso J-R. Nature 2001;410:667.

[36] Kaplan JL, Yorke JA. In: Peitgen HO, Walther HO, editors. Functional differential equations and approximation of fixed points. Berlin: Springer; 1979. p. 20-4. 